\title{
Carotid endarterectomy for severe symptomatic carotid stenosis
}

\section{Endarterectomía carotídea en estenosis carotídea grave sintomática}

\author{
Amado Jiménez-Ruiz¹, Alejandro Gutiérrez-Castillo², and José L. Ruiz-Sandoval ${ }^{3}$ \\ ${ }^{1}$ Department of Neurology, Instituto Nacional de Ciencias Médicas y Nutrición Salvador Zubirán, Mexico City; ${ }^{2}$ Escuela de Medicina, Instituto \\ Tecnológico y de Estudios Superiores de Monterrey, Monterrey, N.L; ${ }^{3}$ Department of Neurology, Hospital Civil de Guadalajara "Fray Antonio Alcalde", \\ Guadalajara, Jal., Mexico
}

An 86-year-old male patient with a history of smoking, dyslipidemia, and hypertension presented with three episodes of motor aphasia of $<1 \mathrm{~h}$ in duration, in the preceding 2 weeks, consistent with a recurrent transient ischemic attack (TIA, 4 points on $A B C D^{2}$ score). Brain magnetic resonance imaging (MRI) showed no evidence of acute infarction, and we started him on dual antiplatelet therapy (aspirin + clopidogrel). The electrocardiogram and the transthoracic echocardiogram showed no valvular or rhythm anomalies. Computed tomography (CT) of the neck revealed a calcified $27 \times$ $8.2 \mathrm{~mm}$ plaque in the left carotid artery with calculated stenosis of $70 \%$ using the North American Symptomatic Carotid Endarterectomy (NASCET) Trial method (Fig. 1). Carotid Doppler study revealed an increased systolic velocity $(464 \mathrm{~cm} / \mathrm{s}$, normal $<125)$. The patient underwent carotid endarterectomy (CE). (Fig. $1 \mathrm{~B}$ and $\mathrm{C}$ ) with longitudinal arteriotomy and patch angioplasty with Dacron. The patient recovered uneventfully and at 1-year follow-up remains symptom free.
$\mathrm{CE}$ is a surgical procedure that seeks the mechanical removal of atheromatous plaques to restore normal blood flow. Patients should be stratified according to the percent of stenosis and the presence or absence of neurological symptoms ${ }^{1,2}$. This procedure is an effective secondary prevention strategy in reducing the risk for infarction in patients with large vessel disease who present with TIA or acute ischemic stroke and have a luminal narrowing of $\geq 70 \%$ using non-invasive imaging studies (CT, MRI, or Doppler ultrasound) or $\geq 50 \%$ on catheter angiography ${ }^{3}$. For men (but not women) with recently symptomatic carotid stenosis of $50-69 \%$ CE can also be considered a therapeutic option ${ }^{3}$.

\section{Funding}

None.

\section{Conflicts of interest}

The authors declare that they have had no conflicts of interest.
Available online: $23-02-2021$ Arch Cardiol Mex. 2021;91(1):123-124 www.archivoscardiologia.com license (http://creativecommons.org/licenses/by-nc-nd/4.0/). 


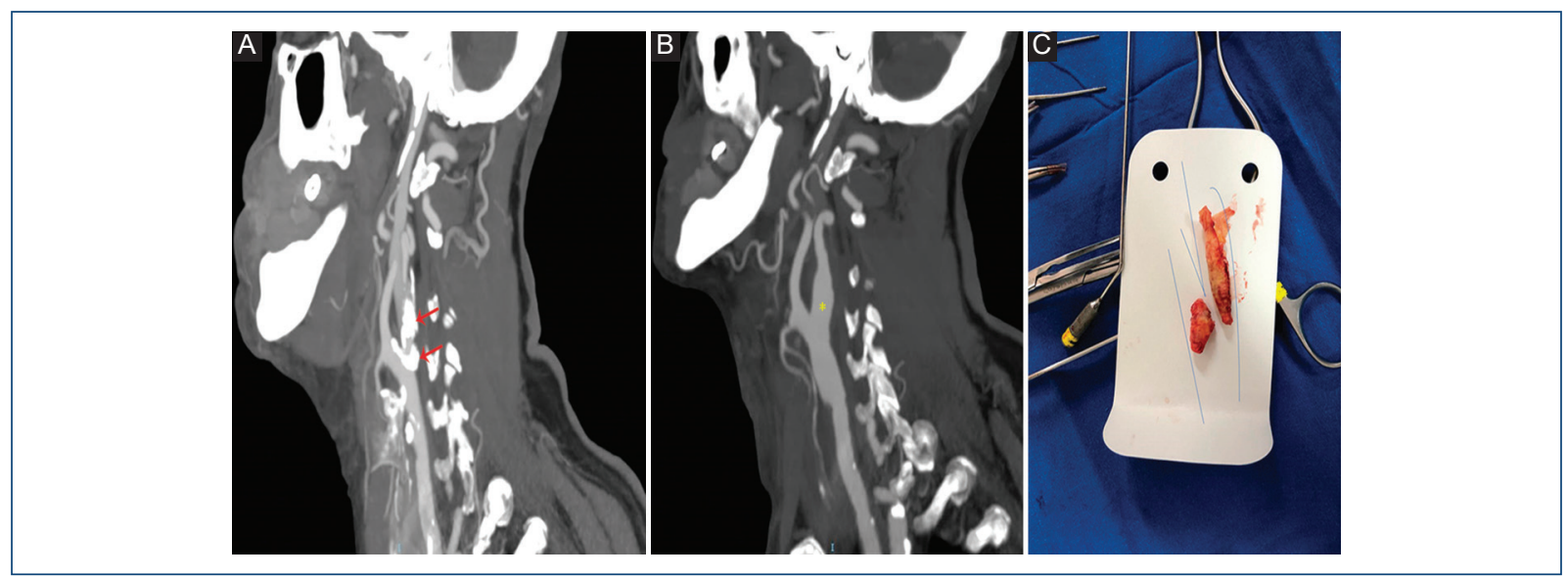

Figure 1. A: computed tomography of the neck shows the presence of two calcified atheromatous plaques (red arrows) at the bifurcation of the left common carotid. B: their absence is shown after carotid endarterectomy (yellow asterisk). C: Gross image of both plaques after their removal.

\section{Ethical disclosures}

Protection of human and animal subjects. The authors declare that no experiments were performed on humans or animals for this study.

Confidentiality of data. The authors declare that they have followed the protocols of their work center on the publication of patient data.
Right to privacy and informed consent. The authors declare that no patient data appear in this article.

\section{References}

1. Waters MF. Surgical approaches to stroke risk reduction. Continuum (Minneap Minn). 2020;26:457-77.

2. Amarenco P. Transient ischemic attack. N Engl J Med. 2020;382:1933-41.

3. Clissold B, Phan TG, Ly J, Singhal S, Srikanth V, Ma H. Current aspects of TIA management. J Clin Neurosci. 2020;72:20-5. 